\title{
On automatic assessment and conceptual understanding
}

\author{
AntTI RASILA $\dagger$, JARMo MaLinen AND HANnU TIITU \\ Aalto University, Department of Mathematics and Systems Analysis, \\ P.O. Box 11100, 00076 Aalto, Finland
}

[Received on X April 2015; revised on Y June 2015]

\begin{abstract}
We consider two complementary aspects of mathematical skills, i.e., procedural fluency and conceptual understanding, from a point of view that is related to modern e-learning environments and computer based assessment. Pedagogical background of teaching mathematics is discussed, and it is proposed that the traditional book medium has determined much of its historical development, including the classical style of presenting mathematical knowledge. Information technology is likely to be an emerging game changer in learning and teaching of mathematics, and we argue that the potential of e-learning platforms extends beyond simple drill exercises to complex problems (having several "right" solutions) that are expected to improve conceptual understanding. We discuss these ideas referring to experiences and observations from a STACK based automatic assessment system at Aalto University since 2006. The article is concluded by visions for the future development involving, e.g., learning analytics based on students' answer data.
\end{abstract}

Keywords: automatic assessment; conceptual understanding; learning games; learning analytics.

\section{Introduction}

During the last 40 years, high density micro-electronics has revolutionised the world we live in, and we still struggle to comprehend the full extent of change. The technological revolution has created new branches of industry such as information and communication technology (ICT), and it has rendered obsolete some other businesses. The impetus of fast computing devices has been powerful enough to shock national economies and even shake the balance of power in international relations. Computers have also affected a particular subject matter that lies in the very foundation of the technological breakthrough while it is, at the same time, at least as old as the western civilisation itself. We refer, of course, to learning and teaching of mathematics.

The purpose of this article is to sketch a brief synthesis of the present state of affairs and some future visions of computerised learning environments for school and university mathematics. We use the report of Kilpatrick et al. (2001) as a background. It has been proposed there that mathematical skills consist of five interrelated fields:

1. Conceptual understanding; i.e., understanding mathematical concepts, operations and relations.

2. Procedural fluency; i.e., the ability to use mathematical procedures such as simplifying formulas.

3. Strategic competence; i.e., the ability to formulate and solve mathematical problems.

4. Deductive abilities; i.e., the ability to think logically, reflect, explain, and prove statements.

${ }^{\dagger}$ Corresponding author. Emails: firstname.lastname@aalto.fi 
5. Interest; i.e., seeing mathematics as meaningful, important, and sensible.

Of these fields, procedural fluency has been emphasised in traditional school teaching (at least) in Finland. It is a clear distinction between school and university levels that deductive abilities play a much greater role in university teaching. For example, proofs of theorems are often not encountered in school mathematics (Joutsenlahti, 2005). The three remaining fields, however, are particularly important for engineers and scientists who should be able to recognise and treat various mathematical problems arising from science and technology. The conceptual understanding is the key element that makes the mathematical problem solving ability truly transferable.

The outline of this article is as follows. Advantages and disadvantages of traditional mathematics teaching are discussed in Section 2. We use there the essay of Richard Skemp (1976) as a main pedagogical background, and this choice leads to an emphasis on conceptual understanding as opposed to focusing on "instrumental" competencies such as procedural fluency. The role of digital learning environments in future mathematics teaching is discussed in Section 3 in the style of Devlin (2011). Sections 4 and 5 are devoted to presenting aspects and observations that have been obtained using the e-assessment system STACK described in Sangwin (2013). We conclude the article by briefly reviewing in Section 6 how some of these aspects relate to Skemp's original vision that predates the digital age.

\section{Learning a mathematical concept}

Unlike in many other fields of education, learning mathematics requires a fair amount of communication with lower level abstractions. In addition to having a foundation of already known mathematical concepts, learning a new concept requires practicing on a suitable collection of examples. This is the way a student becomes familiar with the new concept and is able to either assimilate or accommodate it (Piaget, 1929). Assimilation means that the new information is associated with some of the student's existing conceptual structures, schemas. If the earlier conceptual structures do not allow assimilation, then the schema may change so that the new piece of information fits in - this is accommodation. For example, the mathematical concept of a number is in early childhood understood merely as a naive measure of quantity, but the concept becomes finally a part of an algebraic structure in the course of learning. Within such structure, it is then possible to embrace increasingly more abstract objects: negative numbers, infinite decimals, complex numbers, and so on (Skemp, 1987).

The schematic learning process described above is constructivist (as opposed to behavioristic) since the student's own mental processes are assumed to play the leading role in forming the new concept. The teacher's role is to act as a facilitator and to create favourable conditions for learning. We point out that there are several ways to conceptualise the different levels or aspects of how humans understand mathematics. In Tall (2008), understanding mathematics has been is divided into conceptual-embodied, proceptual-symbolic, and axiomatic-formal worlds. The concepts of instrumental and relational understanding have been used in Skemp (1976), of which "relational" refers, broadly speaking, to the conceptual understanding introduced in Section 1.

How do these ideas relate to the tradition of teaching mathematics? Mathematical knowledge is usually presented in textbooks. While mathematical notation has certainly evolved during centuries, the idea of the textbook itself has seen surprisingly little progress; see Figs. 1 and 2. The formal style

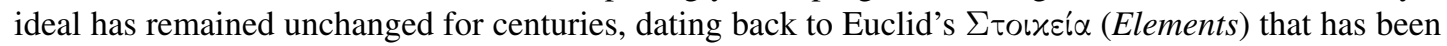
described somewhat polemically as "pedagogically appalling". Indeed, Euclid's axiomatic treatise on geometry gives never any motivations, applications, illuminating examples, or not even cross references except one; see Bochner (1981) but also Kutateladze (2006). 


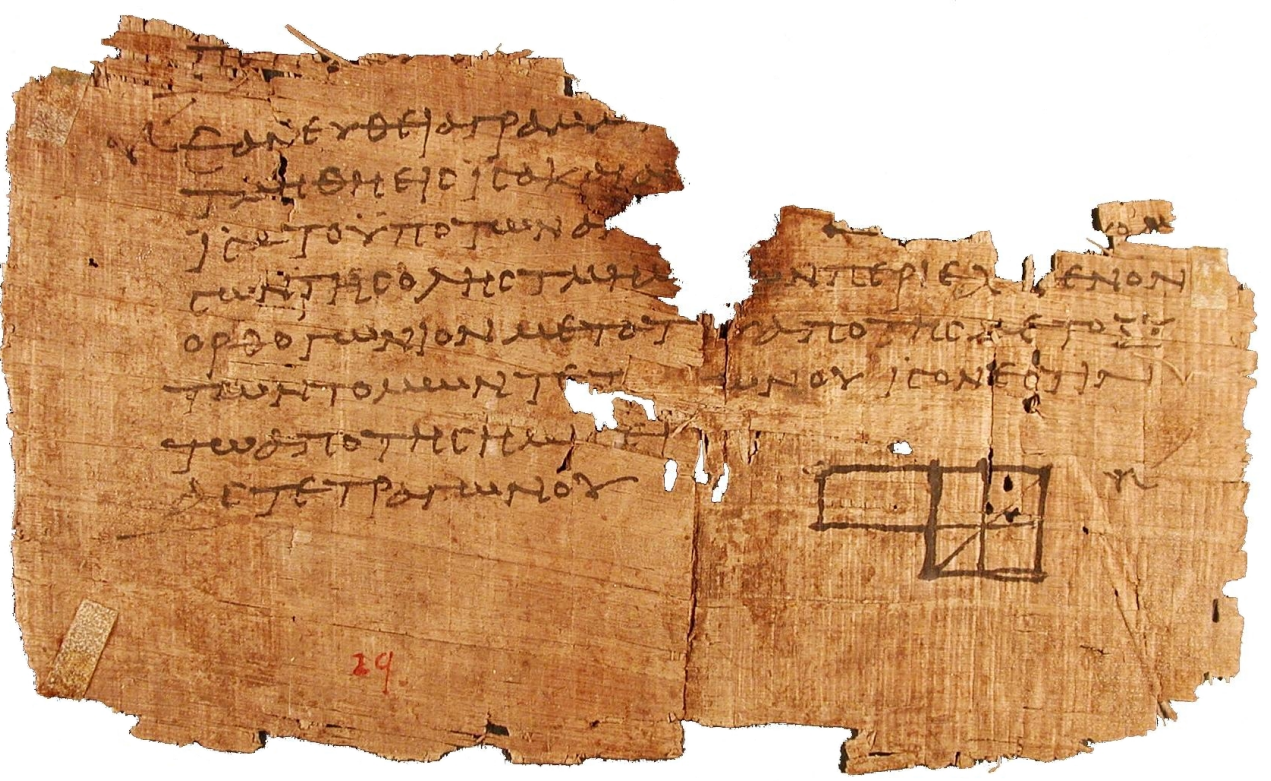

FIG. 1. Papyrus Oxyrhynchus 29, one of the oldest surviving fragments of a copy of Euclid's Elements, currently at the Museum of Archaeology and Anthropology of the University of Pennsylvania. Photo by W. Casselman (used by permission). The original photo can be found at http://www.math.ubc.ca/\%7Ecass/Euclid/papyrus/.

The formalistic philosophy in mathematics education, advocated by some mathematicians, considers the extreme "bourbakist" style as the only right way to present mathematics (see, e.g., Kutateladze, 2006). We see the following as the main shortcoming in the formalistic approach: it focuses only in two of the five aspects of mathematical proficiency mentioned in Section 1, namely, procedural fluency and deductive reasoning. In some modern textbooks, the role of deductive reasoning seems to be much reduced which results in even stronger emphasis on procedural skills.

\section{Teaching mathematical concepts in the digital age}

The exposition in most mathematics textbooks is geared towards efficient preservation and objective transfer of knowledge. Such style may fail to be optimal from the pedagogical point of view, and the problem becomes even more serious in computer assisted learning since the human teacher is likely not to be always present for immediate assistance. The digital study material should stand on its own feet, and hence the requirements are unavoidably higher compared to textbook based learning in classrooms. We conclude that advanced e-learning materials (such as automatically assessed exercises) cannot be successfully developed simply by replicating the traditional teaching media in a computer based learning environment.

There is another serious limitation in digital textbooks and exercise assignments that imitate the traditional pattern. The old ways of presenting mathematics suffer from inevitable technological limitations due to printing press and book format in general, and these limitations may easily but unfortunately get inherited by digital study materials. Such materials make exclusive use of what could earlier be produced easily on paper: formulas, symbols, two-dimensional illustrations, and verbal descriptions of 
8) Zeichne mit dem Lineal eine gerade Linie! Bezeichne auf dieser geraden Linie durch einen kleinen senkrechten Strich einen Punkt A. Nimm $4 \mathrm{~cm}$ in die Zirkelspanne! Setze die Spitze des Zirkels in den Punkt $A$ und schlage einen kleinen Kreisbogen, der die gerade Linie schneidet. Den Schnittpunkt nenne B! Die gerade Linie zwischen den Punkten A und B nennen wir eine Strecke. Sie ist auf beiden Seiten begrenzt.

FIG. 2. The construction of a line segment in elementary geometry. The excerpt is taken from the book Von 1 bis 1000 , Volk und Wissen volkseigener Verlag, 1965.

mathematical ideas. There is no dynamical content, and relations are presented through formulas or algorithms, sometimes supplemented with examples and illustrations of the same kind.

Mathematics is rife with situations where describing intuitively understandable phenomena leads into an almost incomprehensible jungle of definitions. This style of exposition is not entirely without merit since it is quite well suited for preservation of knowledge and for efficient and precise communication between professionals. Similarly, engineers must be able to read and write specifications, protocols, and data-sheets, and the analogous can be said about medical doctors, legal professionals, to mention a few examples of higher professions. We conclude that skills for dealing with complicated definitions is and will always remain an important goal in learning higher mathematics. We argue, however, that it should not necessarily be the means — or, at least, not the only means — of communicating mathematics to students.

Mathematics, if written solely as a collection of rules, is not at all conducive to the "natural" way of understanding observations and underlying relations through trial and error. Such experiments make it possible to see the consequences of small variations to the initial setting, too. All this helps students to get familiar with general ideas and the "big picture" which is often lost, together with the student's motivation, in a jungle of definitions and complicated formulas.

Putting aside many difficult problems in the co-evolution of language and symbolic intelligence in humans, it is safe to assume that our abilities in symbolic intelligence are evolutionarily speaking of rather recent origin. This may well be related to the observation that mathematical ideas are often easier to understand when they are not presented in a purely symbolic form, rather than by using a representation where, e.g., spatio-visual and motoric abilities are advantageous. In fact, professional mathematicians use such "imprecise" representations most of the time when discussing research problems with each other, even though they sometimes need resort to exact definitions and proofs. This observation contradicts the claim that formalism is always the best way of communicating or teaching mathematics.

During the last ten years, Keith Devlin (Stanford University) has introduced a radically different vision for presenting mathematics using modern computers and learning environments. One of Devlin's ideas is using games for presenting mathematical concepts and ideas. The main objective of his research has been to understand how new technologies may affect the development of mathematical knowledge in general, and mathematics learning and teaching in particular (e.g., Devlin, 2011). It is one of Devlin's key observations that traditional mathematics teaching is mostly a legacy from using printed books that 
are full of symbols, formulas, and definitions. Now, there is nothing inherently mathematical in symbols written on paper, and we use them only because we do not know a better way.

Presenting dynamical content is not much of a technological problem in the digital age. The new possibilities of the digital medium allow development of study materials that are more intuitive and informative, resulting in more efficient learning. In addition to the advanced digital content, the technology platform may also allow for intelligent interaction with the student, at least, to some extent. An example of this is provided by automatic assessment systems that are able to classify and diagnose students' answers, give customised feedback, and mark the exercises. It is likely that some future study materials would look nothing like a textbook but more like entertainment products such as video or computer games. According to Devlin (2008), the next revolution in mathematics will change the means of presenting mathematical content rather than the mathematical content itself.

\section{Presenting mathematics in e-learning environments}

Generally speaking, designers of e-learning environments specifically for mathematics are aware of the challenges and the opportunities discussed in Sections 2 and 3. It is also well known that the interaction between the student and the e-learning environment leads to (at least) two kinds of complications that we discuss next.

Firstly, the student may have difficulties in typing in the solution using the computer syntax. The formal language of mathematics is not easy to write in the linear fashion as required by many e-learning environments (Sangwin \& Ramsden, 2007). In the worst case, the student may have to correct mismatching parentheses, add missing multiplication signs, etc., many times until a syntactically valid expression is obtained. The average student is not a computer expert which adds frustration when the system does not respond in the way the student expects. The learning environment may even stress the student and turn the learning opportunity into a meaningless struggle with the "silly computer".

Secondly, mathematical content displayed on computer screen may seem to have some restrictions which partly stem from a narrow, textbook centric point of view. One could also argue that these limitations are, to a significant extent, a plain consequence of copying the style of exposition from printed materials and the associated teaching philosophies. However, purely technical restrictions are becoming less serious due to advances in mathematics display engines such as MathJax (see MathJax Consortium, 2015).

From teachers' and material designers' points of view, the possibilities and the restrictions of the environment affect the construction of the pedagogical and technological framework in which student's various learning processes are to be activated (Majander \& Rasila, 2011; Rasila et al., 2010). For building a pleasant user experience, the presentation of mathematics is in a key role. Mathematical content should be easy to understand even without teacher's help, and the interaction between the student and the computer should be made as effortless as possible. It is quite difficult for the material or system developer to foresee which components or functionalities will eventually cause most negative user experience in students and teachers. To alleviate these problems, it may be beneficial to use modern service design concepts in the organisation of the development work (see, e.g., Stickdorn \& Schneider, 2011). Moreover, some of the problem points in materials and systems may later be detected indirectly, based on students' exercise answer data that gets collected by the e-learning environment. In all cases, user feedback and subsequent revisions of both the e-learning environment and the study materials are required for improving the user experience.

To obtain high quality e-learning materials, it is a good idea to have production teams where both pedagogical and software expertise are equally well represented. We point out that a multi-stage STACK 
TABLE 1. The usage of STACK at the large BSc level engineering mathematics courses at Aalto University during the academic year 2014-15. STACK versions 1 and 3 have been used as shown.

\begin{tabular}{lccccr}
\hline Topic & Version & Courses & Exercises & Students & Marks \\
\hline Matrix algebra & 1 & 7 & 122 & 796 & 16506 \\
& 3 & 1 & 21 & 40 & 679 \\
\hline Single variable calculus & 1 & 4 & 51 & 721 & 11427 \\
\hline Multivariable calculus & 1 & 4 & 48 & 705 & 15536 \\
\hline Vector analysis & 3 & 3 & 42 & 226 & 2748 \\
\hline Probability and statistics & 1 & 2 & 39 & 563 & 12691 \\
& 3 & 2 & 84 & 452 & 14294 \\
\hline Total & & 23 & 407 & 3503 & 73881
\end{tabular}

exercise with full randomisation of parameters may require several dozens of hours development work. Thus, busy university teachers cannot be expected to produce e-learning material apart from simple drills or multiple choice exercises.

We conclude that e-learning environments provide opportunities to do many things that cannot easily, or at all, be done within traditional teaching, and this is where e-learning methods are at an advantage. By no means are e-learning environments expected to match the traditional teaching where traditional methods work best, and nothing stops the teacher from using lectures and textbooks together with e-learning materials.

\section{Automatic assessment at Aalto University}

The computer aided assessment system STACK was introduced at Helsinki University of Technology (nowadays part of Aalto University) in 2006. STACK is GPL-licenced open source software, originally developed by Chris Sangwin in University of Birmingham. It is an e-learning environment that is suitable for developing automatically assessed exercise assignments that contain mathematical symbols, formulas, and concepts. STACK consists of a computer algebra system for evaluating symbolic expressions, a web-based user interface (currently Moodle), and a database for storing exercise assignments and students' solutions. The system allows various pedagogical approaches and assignment types ranging from simple drills to sophisticated simulation and modelling problems that may have several valid solutions.

The STACK system has been adapted for the requirements of engineering mathematics courses at Aalto University (Sangwin, 2013). Numerous new features have been implemented to widen the applicability and to improve the human-computer interaction. These features included both user input and display modifications such as multiple input fields in one exercise, two dimensional (i.e., matrixvalued) input fields, and more browser independent rendering of mathematical formulas and answer dialog elements (see Harjula, 2008). Examples of more recent development are allowing output of variables in Maxima syntax, and the conditional and iterative generation of question text. The latter includes introducing if and foreach expressions to STACK syntax, the possibility of re-defining of variables inside the question text, and content generation with external tools using parameters from the 

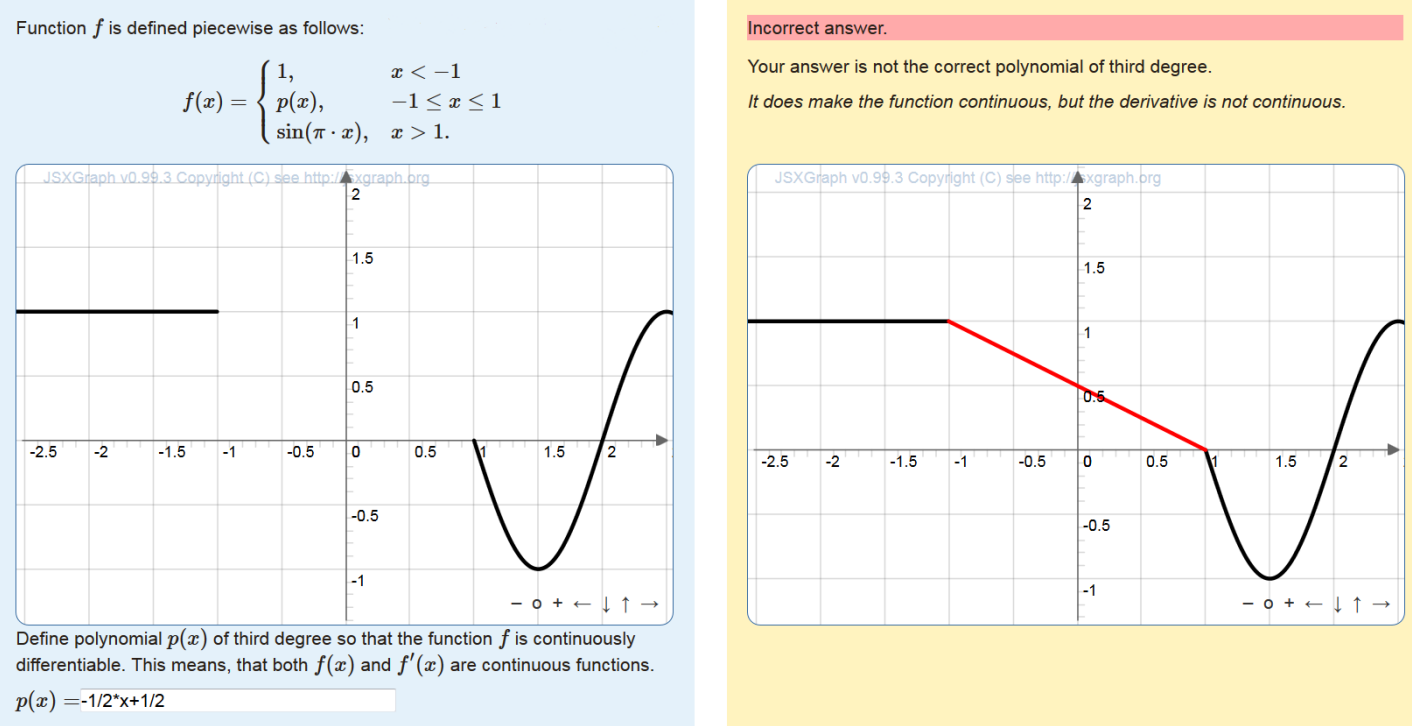

FIG. 3. Left panel: Dynamically generated image within a STACK exercise. Right panel: The visualisation of the student's answer in the feedback given by STACK.

question or student's answer to it. The development branch of Aalto will be fully merged into the main trunk of the codebase at the publication of the next release of STACK.

At the time of writing of this article, there are STACK exercises in practically all BSc level courses of engineering mathematics at Aalto. The volumes of the large courses are shown in Table 1. These courses are intended for the first and the second year students from various fields of engineering and science. STACK is also used in more advanced courses such as complex analysis and discrete mathematics, and for teaching other mathematical subjects such as physics and logistics (not shown in Table 1).

At Aalto, the BSc level mathematics courses consist of lectures, classroom exercises, and STACK exercises. These courses last six weeks, and students receive each week up to ten STACK exercises. STACK replaces some of the traditional classroom exercises as a form of continuous assessment since the final grades are a result of the mid-term exams and the points earned in the exercises. Currently, two versions of STACK are in use. Version 1 is the customised version of STACK 1 developed at Aalto, and version 3 refers to the latest Moodle based release of STACK (see Harjula, 2008; Sangwin, 2013). The student user numbers for these versions are 1629 and 1156, respectively, during the academic year 2014-15.

The observed benefits from using an automatic assessment system comparable with STACK include the following.

1. Compared to traditional classroom teaching, STACK provides additional flexibility and saves time for the students and the teachers alike (Rasila et al., 2007). The saved time can be used to provide additional student support.

2. Typically, a STACK assignment produces for each student an individualised version of the same exercise that, however, is of comparable degree of difficulty. The students can then work together 
without the risk of just copying the solutions from each other. Working together encourages peer learning and discussions about the underlying concepts (Rasila et al., 2010).

3. The feedback is essential in guiding the student's learning process. The immediate feedback was found to be the most popular feature of STACK by students as reported in Sangwin (2013, Section 7.10). In STACK exercises, feedback can be made detailed enough to help the student to overcome misunderstandings or mental hang-ups (Rasila et al., 2010). This, however, requires deep analysis of students' solution processes which is a challenging task.

4. Using STACK, the grading can be based on continuous evaluation of the work the student has been doing during the semester, instead of a short list of examination problems. This can improve content validity, reliability, and fairness of the grading, and lead to more balanced workload through the duration of the course (Majander \& Rasila, 2011).

5. STACK assignments may contain dynamically generated images and other interactions. The interactive visualisations can be used to show the student why the solution is right or wrong. As an example of this, Fig. 3 is used to concretise the concept of continuous derivative. Student was asked to extend a given function so that the extended function becomes differentiable on the larger domain. Visual feedback from STACK plots student's incorrect answer and the discontinuity of the derivative can be seen at the ends of the interval $[-1,1]$, and the graph is accompanied by a written explanation.

6. The STACK system can also be used as a programming environment for developing simple elearning games that have potential to make the learning process more meaningful, motivating, and interactive. Example in Fig. 4 shows an exercise where the student is asked to evaluate different risk scenarios in running a business in an unstable environment. The last part of the exercise is generated according to student's answer of the previous part, thus affecting the progression of the storyline.

7. Performance and progress can be evaluated by analysing student's errors that have been logged by STACK. One way of doing this is manual or automatic error categorisation according to a predefined classification model. The classification model should be objective and validated using statistical techniques (see, e.g., Dahl et al., 2014).

In addition to drilling styled exercises we have implemented some quite simple game-like series of assignments where the individual assignments, however, cannot share information with each other due to restrictions of current version of STACK. In other words, the expression "game-like" should here be understood as follows: a logically ordered series of exercises with a common plot. It is certainly possible to further develop STACK to enable more advanced game-like or even gamified learning. Then the individual assignments could be interconnected into highly interactive storylines describing interesting real world applications. In the current version of STACK, the selection of assignments cannot be customised based on student's past performance or observed learning style, and neither can the behaviour of an individual STACK assignment depend on the past performance of the student or student's peers in earlier assignments. 
Before answering these questions, please get familiar with the background material.
Answer first parts $A$ and $B$, then press the Check-button before answering the final part C.

Part A. Evaluate the following risks.

(risk $=$ probability of an unwanted event per year $\times$ damage costs in euros)

Use the probabilities on page 2 of the background material.

Round risks to the closest million. Give the answer in whole millions, i.e. 5 means 5 million euros.

a) A serious fire accident at the factory. $50 \%$ of the business premises and equipment is destroyed, and the operations are closed down. The annual turnover decreases by $50 \%$. Risk: 3 M€

b) 5 of the employees kidnapped. Risk: 5 M€

c) The factory is closed down as a consequence of an earthquake.

There are no casualties but the annual turnover decreases by $20 \%$. Risk: 6 M€

d) Corrupt officials claim that the machinery does not satisfy work safety regulations.

The factory is closed down. Risk: 3 M€

Part B. Choose one of the risk protection measures presented in the background material.

1) Property \& Business Interruption Insurance, 2) Bribing, 3) Recruiting a Security Manager,

or 4) Kidnapping \& Ransom Insurance. Enter number 1-4 and press the Check-button. Your choice: 3

Part C. According to your choice in part B, your risk scenario is following.

Selected protection measure is 3: Recruiting a Security Manager.

After six months of production, 5 of the employees are kidnapped.

Calculate the net profit after the incident: $\square$ M€

Check

FIG. 4. A game-like STACK exercise in three parts. The scenario for the last part is generated according to student's choice in the previous part.

\section{Improving mathematical competency with computers}

We conclude by discussing how an automated assessment system could help in improving and evaluating advanced mathematical competencies such as conceptual understanding.

While repetition and drilling are the core of all instrumental and rote learning, this is by no means the only possible use for such exercises: repetition plays an important role in constructivist pedagogics as well. According to Skemp (1987), to understand a mathematical concept it is important to run through it many times. It is essential that the required elementary cognitive processes become automatic, thus freeing the student's attention to concentrate on the new ideas that are being learnt. Eventually, mathematical symbols get detached from their concepts, and manipulating them can be performed without paying attention to their meaning. All this requires usually a lot of practice. While there is nothing e-learning specific in these observations by Skemp as such, computer aided visualisations and experimentations can obviously be quite valuable in facilitating the learning process described above.

How do we know the amount of practice required, or if any progress has taken place? One possibility is to analyse the large amount of data, produced by the e-learning environment, to track and optimise the learning process. Some steps in this direction were taken in Linnoinen (2013) where the Granger causality was used for testing the hypothesis that working more leads to better success in later math- 
ematics studies. Development of conceptual understanding was being tested in this work rather than instrumental skills (such as procedural fluency) because the students were required to learn completely new skills. The ability to learn these skills was then reflected against students' background.

From the point of view advocated by Skemp $(1987,1976)$, the most important goal of teaching mathematics is the cognitive development of the student. Transfer and construction of factual knowledge should be seen as tools for achieving it. Students' motivations and attitudes, time available for learning, and too large teaching groups may hinder the sought-after cognitive development. Many students wish for immediate benefits of what they just have learned, and they are often frustrated by excessive work and perceived difficulty. For this reason, it is worthwhile to give the students relatively easy problems which encourage many of them to think, rather than deep problems which give satisfying intellectual challenge for the few and discourage the others. It is very difficult for the teacher to appraise even the most basic matters such as the sufficient amount of work required for learning, and what is too challenging for most of the students. There are large differences between students taking the same course, and it is practically impossible to cover their individual needs in traditional classroom teaching. Diagnostic testing can be used to set the right starting level for a course, but there is often not much besides teacher's experience to guarantee the success.

In principle, e-learning environments could produce individualised exercise assignments that are on the right level for each student, and they could even help students in finding appropriate study material to overcome their individual weaknesses. STACK stores large amounts of students' answer data which contain patterns that can be found by data mining techniques. A software agent could be designed for the error classification based on full answer data instead of using, e.g., fixed diagnostic exercises for semi-manual student profiling. Based on the profiles, randomised and personalised additional study materials can be automatically produced for each student. While this level of sophistication is beyond the capabilities of currently available e-learning environments, we expect tools for such purposes to be developed in the future.

\section{REFERENCES}

Bochner, S. (1981) The Role of Mathematics in the Rise of Science. Princeton: Princeton University Press.

DAhl, B., StÅhl, T., Malinen, J., Rasila, A. \& Tittu, H. (2014) Diagnosing nursing students' errors in medication calculation. Designing a method based on the $4 \mathrm{Cs}$ teaching model for analysing mathematical proficiency. In Viteli, J. \& Östman, A. (eds.) Tuovi 12: Interaktiivinen tekniikka koulutuksessa 2014 konferenssin tutkijatapaamisen artikkelit. TRIM Research Reports 12. Tampere: University of Tampere, pp. 82-92. Available at http://urn.fi/URN:ISBN:978-951-44-9561-8

DeVlin, K. (2008) What will count as mathematics in 2100? In Gold, B. \& Simons, R. (eds.) Proof and Other Dilemmas: Mathematics and Philosophy. Mathematical Association of America, pp. 291-311.

DEVlin, K. (2011) Mathematics Education for a New Era. Video Games as a Medium for Learning. Natick, MA: A K Peters.

HARJUla, M. (2008) Mathematics exercise system with automatic assessment. Master's thesis. Helsinki University of Technology. Available at http://urn.fi/URN:NBN:fi:aalto-201306116486

JoutSENLAHTI, J. (2005) Lukiolaisen tehtäväorientoituneen matemaattisen ajattelun piirteitä. In Finnish. PhD thesis. Acta Universitatis Tamperensis 1061. University of Tampere.

Kilpatrick, J., Swafford, J. \& Findell, B. (eds.) (2001) Adding it up: Helping children learn mathematics. Mathematics Learning Study Committee, Center for Education, Division of Behavioral and Social Sciences and Education, National Research Council. Washington, DC: National Academy Press.

Kutateladze, S. S. (2006) Apology of Euclid. Scientiae Mathematicae Japonicae/Notices from the ISMS, 4(5), 1-3. 
Linnoinen, K. (2013) Does Practice Make Perfect? A Study of the Granger-causal Relationship Between Attempting to Solve Online Exercises and Mathematical Proficiency. Master's thesis. University of Helsinki. Available at http://hdl.handle.net/10138/42205

MAJANDER, H. \& RASILA, A. (2011) Experiences of continuous formative assessment in engineering mathematics. In Silfverberg, H. \& Joutsenlahti, J. (eds.) Integrating Research into Mathematics and Science Education in the 2010s. Annual Symposium of the Finnish Mathematics and Science Education Research Association 14.-15.10.2010 in Tampere. Tampere: Juvenes Print, pp. 197-214.

Available at http://matta.math.aalto.fi/publications/MajanderRasila2011.pdf

MathJax Consortium. (2015) MathJax: Beautiful math in all browsers. Available at https://www.mathjax.org [Accessed: 29th May 2015]

Piaget, J. (1929) The Child's Conception of the World. London: Routledge \& Kegan Paul.

Rasila, A., Harjula, M. \& Zenger, K. (2007) Automatic assessment of mathematics exercises: Experiences and future prospects. In Yanar, A. \& Saarela-Kivimäki, K. (eds.) ReflekTori 2007. Symposium of Engineering Education, December 3-4, 2007. Helsinki University of Technology Teaching and Learning Development Unit Publication 1/2007. Espoo: Helsinki University of Technology, pp. 70-80. Available at http://matta.math.aalto.fi/publications/Reflektori2007_70-80.pdf

Rasila, A., Havola, L., Majander, H. \& Malinen, J. (2010) Automatic assessment in engineering mathematics: evaluation of the impact. In Myller, E. (ed.) ReflekTori 2010. Symposium of Engineering Education, 9-10 December 2010, Espoo, Finland. Dipoli-Reports B 2010:1. Espoo: Aalto University, pp. 37-45. Available at http://urn.fi/URN:ISBN:978-952-60-3478-2

SAngwin, C. (2013) Computer Aided Assessment of Mathematics. Oxford: Oxford University Press.

SANGWIN, C. J. \& RAMSDEN, P. (2007) Linear syntax for communicating elementary mathematics. Journal of Symbolic Computation, 42(9), 920-934.

SKEMP, R. R. (1976) Relational Understanding and Instrumental Understanding. Mathematics Teaching, 77, 20-26.

SkEMP, R. R. (1987) The Psychology of Learning Mathematics. Expanded American Edition. New York: Routledge.

Stickdorn, M. \& Schneider, J. (eds.) (2011) This is Service Design Thinking: Basics - Tools - Cases. Hoboken, NJ: Wiley.

TALL, D. (2008) The transition to formal thinking in mathematics. Mathematics Education Research Journal, 20(2), 5-24. 